\title{
Literature Review on R\&D Innovation of Family Firms
}

\author{
Yiqing Yang ${ }^{1, a,}$ Shouye $\mathrm{Liu}^{2, \mathrm{~b}, *}$
}

${ }^{I}$ Zhejiang Merchants Research Institute of Zhejiang Gongshang University, Hangzhou, Zhejiang, China

${ }^{2}$ School of Business Administration, Zhejiang Gongshang University, Hangzhou, Zhejiang, China

a1530729444@qq.com

b*1083948557@qq.com

\begin{abstract}
Research on family firm's innovation is an important topic in the field of family firms research. First of all, the definition of family firms includes element participation method, essential definition method and continuous definition method. The second is to integrate relevant theoretical tools in this field, including agency theory, stewardship theory and social emotional wealth theory. Finally, the paper analyzes the double-layer factors that influence the R\&D innovation of family firms.
\end{abstract}

Keywords: family firms, $R \& D$ innovation, $S E W$

\section{关于家族企业研发创新的文献综述}

\author{
杨轶清 $1, \mathrm{a}$ 刘寿叶 $2, \mathrm{~b}, *$
}

${ }^{1}$ 浙江工商大学浙商研究院, 杭州, 浙江, 中国

${ }^{2}$ 浙江工商大学工商管理学院, 杭州, 渐江, 中国

a1530729444@qq.com

b*1083948557@qq.com

\section{摘要}

家族企业创新研究是家族企业研究领域的重要议题, 通过对以往文献梳理整合了这一领域的相关研究。 首先是对家族企业概念的界定方法包括要素参与法、本质定义法和连续定义法; 其次是对研究这一领 域的相关理论工具进行整合主要包括代理理论、管家理论和社会情感财富理论; 最后是分析以往文献 中提出的影响家族企业研发创新的双层因素。

关键词：家族企业；研发创新；社会情感财富

\section{1. 家族企业界定}

家族企业在企业组织占据重要地位，在家族企业领 域开展研究, 首先必须完成的是对于家族企业的合理界 定 ${ }^{[1]}$, 这也是目前面临的一大挑战, 如果无法对这一概 念达成一致也就无法对这一领域的相关研究形成统一 意见。目前对于家族企业的定义主要是包括家族要素参 与定义法、本质定义法、连续定义法。首先是参与要素 定义法, 这个方法主要是考察家族在企业里的股权参 与、管理权参与、同时参与以及跨代控制的程度。首先 是从所有权参与角度, 将家族企业定义为是由某个家族 或家族成员控制, 首先是家族企业在所有权层面上对企 业的股权控制超出一定比例, 其次是在管理权上能够参 与企业的管理 ${ }^{\left[{ }^{[2]}\right.}$ 。管理权参与的界定方法受到青睐 ${ }^{[3]}$, 并指出家族企业是家族对企业掌握着绝对的控制权, 这 种控制权包括在董事会上的涉入一一决策权的控制以
及在管理层上的涉入一一管理权的控制。但是随着家族 企业发展进入现代企业制度，其核心是所有权和经营权 的相互分离, 使得对于家族企业的界定需要同时关注股 权和管理权。有学者指出家族企业的能否实现代际传承 也是识别家族企业的重要特征 ${ }^{[4]}$ 。

实际上这种界定家族企业的方法没有关注到家族 企业的可能面临的特殊情景, 于是学者们提出了本质定 义法和连续定义法, 本质定义法通过家族企业的本质去 定义而连续定义法希望打破家族企业以上两种方法的 “二分性”。国内学者李善民、王陈佳将家族企业刻画 为家庭式企业、纯家族企业、准家族企业、混合式家族 企业就是一种连续定义法 ${ }^{[5]}$ 。

\section{2. 相关理论工具梳理}

国内外相关学者对于家族企业的研究借用了不同 的理论工具, 其中较为典型的包括代理理论, 管家理论 
和社会情感财富理论。这三大理论突出的运用是在研究 家族控制和企业绩效的关系之间以及家族企业研发创 新的问题上。

\section{1 代理理论}

代理理论的核心观点是假设资本持有者和经理人 之间职能分工所创造的收益。一方面对于资本持有者而 言他们不需要具备专业的投资管理能力就可以从创业 投资中获益; 另一方面经理人不需要有资本, 可以利用 他们的专业能力抓住有利可图的商业机会 ${ }^{[6]}$ 。但现实情 况是往往出现的不是代理收益而是代理问题, 由于信息 不对称和代理人与委托人之间的利益分歧所带来代理 问题, 而解决这些代理问题所付出的就是代理成本。

对于家族企业而言, 一方面家族的涉入能够解决由 于利益分歧所带来的代理问题, 指出家族涉入管理带来 了所有者和管理者的一致利益, 在企业之间产生一种协 同效应从而降低代理成本形成代理优势。另一方面, 家 族高管也会利用自己的优势地位来剥削小股东, 并以牺 牲公司为代价让自己受益, 通过一些搭便车和逃避行为 抢占公司的创新资源从而对创新产生阻力 ${ }^{[7]}$ 。甚至有学 者认为家族对于所有权和控制权的单方面占有, 这也意 味着家族企业需要独立承担研发创新所带来的风险和 收益, 而投资失败的风险往往直接对整个家族的利益产 生影响, 因此家族企业往往选择一些风险较小, 投资回 报比较快的项目 ${ }^{[8]}$ 。

\section{2 管家理论}

代理理论为管家理论的发展奠定了基础, 管家理论 进一步改善了代理理论对于代理人人性的假设, 管家理 论假定了代理人管理企业是出于实现自我的内心需要, 这使得委托人和经理人之间的利益达成一致, 经理人以 实现家族企业利益最大化为自己的使命和目标 ${ }^{[9]}$ 。对于 家族企业而言, 家族成员更像是一个家族企业的好管 家, 专注于企业的长期利益, 并且与企业内外各种利益 相关者保持亲密的关系, 推动企业的创新和研发活动 ${ }^{[7]}$ 。

家族高管对于公司的依恋和忠诚程度很高, 因为他 们认为企业是家族和个人的情感来源, 是当代和后代经 济支持的载体。因此这些高管会通过对人才的激励和对 利益相关者伙伴关系的慷慨投资来实现自己对企业的 长期管理, 对于企业长期利益的关注使得家族企业更愿 意从事研发投资 ${ }^{[10]}$ 。另一方面, 有学者指出家族高管不 是企业的管理者, 而是直系亲属的管理者, 此时家族高 管可能会给予亲属不属于他们的职位和回报, 这种行为 可能会损害公司利益并阻碍公司的研发创新 ${ }^{[7]}$ 。

\section{3 社会情感财富理论}

家族企业的最本质特质就是重视整体的家族利益。 Gomez-Mejia 等学者在 2007 年首次提出社会情感财富
SEW 的概念, 将家族企业里的非财务目标划入这个概念 里, 并提出相应的家族企业战略决策的模型 ${ }^{[11]}$ 。就家族 企业而言, 对社会情感财富的持有是一项重要的任务, 社会情感财富概括了一个家族从企业获得的情感价值, 社会情感财富里包含着一些共同的家族目标。家族企业 会以 SEW 为决策框架, 家族企业的决策基于社会情感参 照点, 而不仅仅是经济参照点。这也解释了为什么为什 么家族企业看重声誉和重视社会责任的相关活动 ${ }^{[12,13]}$, 即使这些行为在短时间内不会提升企业的财务绩效。

对于 SEW 的进一步研究使得学者们认为家族企业不 愿意进行研发投资 ${ }^{[14]}$, 因为创新是一项需要长期投入的 活动, 创新一旦失败对于家族企业而言将会面临 SEW 和 财务损失的双重风险。实际上, 这些研究缺乏对于 SEW 本身的考察, SEW 本身可以分为 5 各维度包括: 家族控 制、家族成员对企业的认同、紧密的社会关系、情感联 系和跨代控制 ${ }^{[15]}$ 。实际上已有实证研究指出重视跨代控 制型 SEW 的家族企业往往更愿意从事研发投资, 因为这 有助于提升家族企业的长期传承 ${ }^{[10]}$ 。国内学者继续将 SEW 分为约束型和延伸型, 并指出由于外部管理人员的 介入和家族股份的稀释以及特殊性契约的存在使得家 族企业处于约束型 SEW 时会减少研发投资, 而处于延伸 性 SEW 时会增加研发投资 ${ }^{[16]}$ 。

\section{3. 影响家族企业研发创新的内层因素}

国内外对于影响家族企业研发创新因素的研究有 很多, 归结起来主要是包括内部因素和外部因素的双重 作用。其中内部因素主要包括企业治理结构和社会情感 财富。

企业治理结构对于研发创新的影响是家族企业创 新领域研究最多的, 主要的理论工具是代理理论和管家 理论。国外学者 17 以台湾电子行业上市公司为样本将 家族企业与非家族企业进行对比认为家族所有权阻碍 了研发投资, 这可能会阻碍企业创新的推进。此外, 当 CEO - 董事长职位分离或董事会中包含更多独立的外部 人士时, 家族持股比例较高的公司可能会增加研发投资 [17]。之后又通过问卷研究认为家族所有制会对企业的战 略控制具有显著相关，因此对企业创新有显著的影响 [18]。治理结构的研究内容不仅包括股权和管理权上的涉 入, 还包括企业本身规模大小所造成的影响, Gudmundson 通过对于美国中小企业的研究认为中小型 家族企业比非家族企业的创新程度更高 ${ }^{[19]}$ 。国内学者李 婧、贺小刚通过研究认为家族股权集中度过高会对研发 创新产生抑制作用 ${ }^{[20]}$ 。

社会情感财富对于研发创新的影响兴起较晚, 但受 到关注。有学者以代理理论和社会情感财富理论的结合 为工具认为家族企业研发强度会随着期望和绩效的差 距而变化, 尤其在重视跨代控制的家族企业里会增加研 发支出 ${ }^{[10]}$ 。吴炳德、陈凌 21 区分了在家族治理中社会 情感财富和研发投资的不同组合 ${ }^{[21]}$ 。而朱沉等学者对于 社会情感财富本身进行了研究, 认为只有约束型社会情 感才会抑制家族企业的研发创新, 而延伸型社会情感财 


\section{5. 文献评述与研究启示}

综合以上文献可以得出：（1）对于家族企业的统 一界定标准目前还没有能够达成一致的看法, 实际上无 论是采用哪种定义方法基于不同国家和不同情景之下, 对于家族企业样本的选取都是不同的, 这也会对于研究 的结果造成影响。（2）家族企业是不同于其他企业的 一种组织形式, 因为在家族企业中受到两种逻辑的冲突 的影响包括企业逻辑和社会情感财富逻辑, 家族企业在 不同阶段的研发创新差异实际上是这两种逻辑冲突的 结果。（3）对于家族企业影响因素的考量是一个不断 渐进并且深入的过程, 在这个过程中学者们突破了单一 因素达成一致的认为家族企业研发创新实际上是多重 影响因素的整体作用。

通过对这些文献的梳理我们也可以发现家族企业 创新研究领域需要突破的地方：（1）目前对于家族企 业样本的采集主要是通过要素参与法, 通过对家族在企 业中的所有权和管理权去界定家族企业, 这种方法可操 作性强但是存在缺陷, 相比之下本质定义法和连续定义 法是对要素参与法的补充, 但是在目前的研究中应用不 广, 可操作性有限。（2）目前对于家族企业研发创新 上多采取的是实证研究, 并且样本主要选自上市的家族 企业, 样本研究范围的覆盖群体有限, 这也是导致研究 结果出现差异的原因之一。(3) 家族企业创新影响因 素的研究上大部分研究还是采取单一层面上的影响因 素, 纳入其他层面影响因素的研究能够较为全面揭示家 族企业研发创新机制的黑箱, 从而提出更有效的建议。 家族企业作为一种长期存在的组织形式, 在商业社会上 占据重要的地位, 是促进我国国民经济增长的重要力 量, 也是世界经济的重要组成部分。目前在国民经济转 型升级背景下, 家族企业面临着代际传承、治理结构的 调整等难题。创新则是家族企业基业长青和突破困境的 重要途经。未来家族企业创新领域的研究还可以在以下 几个方面进行探索; 首先是在家族企业的界定上, 能否 在更大的范围内达成一致？其次是对家族企业的创新 影响因素上, 关于内层因素的研究已经比较多了, 可以 更多转向外层因素的研究以及内外层因素的共同作用, 完善家族企业创新机制的研究。最后对于创新能力和创 新强度的评鉴上, 以往研究文献里基本上都是使用单个 创新产出或创新投入的指标, 实际上去衡量企业的创新 应综合考虑研发创新投入、过程和产出几个阶段的综合 结果。

\section{References}

[1] Handler, W.C., (1989) Methodological Issues and Considerations in Studying Family Businesses. Family Business Review, 03: 257-276.

[2] Chrisman, J.J., J.H. Chua and L. Steier, (2005) Sources and consequences of distinctive familiness: An introduction. Entrepreneurship: Theory and Practice, 03: 237-247. 
Future Research. Family Business Review, 13: 258-279.

[3] Reeb, R.C.A.A., (2003) Founding-Family Ownership and Firm Performance: Evidence from the S\&P 500. The Journal of Finance, 03: 1301-1328.

[4] C., H.W., (1990) Succession in Family Firms: A Mutual Role Adjustment Between Entrepreneur and Next-Generation Family Members. Entrepreneurship Theory and Practice, 01: 37-51.

[5] Li Shanmin, Wang Chenjia, (2004) The Definitions and Models of Family Enterprise, Journal of Sun Yatsen University Social Science Edition, 44: 66-70.

[6] Munari, F., R. Oriani and M. Sobrero, (2010) The effects of owner identity and external governance systems on R\&D investments: A study of Western European firms. Research Policy, 39: 1093-1104.

[7] Miller, D., A. Minichilli and G. Corbetta, (2013) Is family leadership always beneficial? Strategic Management Journal, 34: 553-571.

[8] Patel, P.C. and J.J. Chrisman, (2014) Risk abatement as a strategy for R\&D investments in family firms. Strategic Management Journal, 35: 617-627.

[9] Le Breton-Miller, I. and D. Miller, (2009) Agency vs. stewardship in public family firms: A social embeddedness reconciliation. Entrepreneurship: Theory and Practice, 33: 1169-1191.

[10] Chrisman, J.J. and P.C. Patel, (2012) Variations in R\&D Investments of Family and Nonfamily Firms: Behavioral Agency and Myopic Loss Aversion Perspectives. Academy of Management Journal, 55: 976-997.

[11] Gómez-Mejía, L.R., et al., (2007) Socioemotional wealth and business risks in family-controlled firms: Evidence from Spanish olive oil mills. Administrative Science Quarterly, 01: 06-137.

[12] Deephouse, D.L. and P. Jaskiewicz, (2013) Do Family Firms Have Better Reputations Than Non-Family Firms? An Integration of Socioemotional Wealth and Social Identity Theories. Journal of Management Studies, 50: 337-360.

[13] Berrone, P., C. Cruz and L.R. Gomez-Mejia, (2012) Socioemotional Wealth in Family Firms. Family Business Review, 25: 258-279.

[14] Chen Jianlin, Feng Xinjun, Li Ruiqin. (2018) Do Family Firms Promote or Hinder Innovation? Debate and Integration, Foreign Economics \& Management, 40: 140-152.

[15] Berrone, P., C. Cruz and L.R. Gomez-Mejia, (2012) Socioemotional Wealth in Family Firms: Theoretical Dimensions, Assessment Approaches, and Agenda for
[16] Miller, D. and I. Le Breton-Miller, (2014) Deconstructing socioemotional wealth. Entrepreneurship: Theory and Practice, 38: 713-720.

[17] Chen, H. and W. Hsu, (2009) Family Ownership, Board Independence, and R\&D Investment. Family Business Review, 22: 347-362.

[18] Hsu, L. and H. Chang, (2011) The Role of Behavioral Strategic Controls in Family Firm Innovation. Industry and Innovation, 18: 709-727.

[19] Gudmundson, D., C.B. Tower and E.A. Hartman, (2003) Innovation in Small Businesses: Culture and Ownership Structure Do Matter. Journal of Developmental Entrepreneurship, 01: 1.

[20] Li Jing, He Xiaogang. (2012) Ownership Intensity and Innovative Performance: A Comparative Analysis between State-Owned and Family-Owned Firms, Journal Of Business Economics, 10: 40-51.

[21] Wu Bingde, Chen Lin. (2014) Socialemotion Wealth and R\&D Investment Portfolio: An Empirical Study of the Impact of Family Governance, Studies in Science of Science, 32: 1233-1241.

[22] Zhu Hang, Eric Kushins, Zhou Yinghui. (2016) Does social emotional wealth inhibit investment in innovation in Chinese family firms? Management World, 63: 99-114.

[23] North, D.C., (1990) Institutions, institutional change, and economic performance. Cambridge University Press, The Cambridge.

[24] Peng, M.W., (2003) Institutional Transitions and Strategic Choices. Academy of Management Review, 02: 275-296.

[25] Chu Xiaoping, Li Huaizu, (2003) Growth Of Family Firms And Merge Of Social Capitals, Economic Theory and Business management, 06: 45-51.

[26] Li, J., J. Xia and E.J. Zajac, (2018) On the duality of political and economic stakeholder influence on firm innovation performance: Theory and evidence from Chinese firms. Strategic Management Journal, 39: 193-216.

[27] Li Jing, He Xiaogang, (2012) Ownership Intensity and Innovative Performance: A Comparative Analysis between State-Owned and Family-Owned Firms, Journal Of Business Economics, 10: 40-51.

[28] Pi Yonghua, Bao Gongmin, (2005) An Empirical Study of the Relationship Between Diversification Strategy and R\&D Intensity of Chinese Firms, Science Research Management, 26: 76-82. 
China's Firm-level Analysis, Journal of Financial Research, 01: 177-190.
[29] Wang Lanfang, Hu Yue, (2017) Does Venture Capital Improve Innovation Efficiency? Evidence from 